\title{
Understanding Intensity Non-uniformity in MRI
}

\author{
John G. Sled and G. Bruce Pike \\ McConnell Brain Imaging Centre, Montréal Neurological Institute, \\ McGill University, Montréal, Québec, Canada
}

\begin{abstract}
Motivated by the observation that the diagonal pattern of intensity non-uniformity usually associated with linearly polarized radiofrequency (RF) coils is often present in neurological scans using circularly polarized coils, a theoretical analysis has been conducted of the intensity non-uniformity inherent in imaging an elliptically shaped object using 1.5 $\mathrm{T}$ magnets and circularly polarized RF coils. While an elliptic geometry is too simple to accurately predict the variations in individual anatomical scans, we use it to investigate a number of observations and hypotheses. (i) The widely made assumption that the data is corrupted by a smooth multiplicative field is accurate for proton density images. (ii) The pattern of intensity variation is highly dependent on the shape of the object being scanned. (iii) Elliptically shaped objects produce a diagonal pattern of variation when scanned using circularly polarized coils.
\end{abstract}

\section{Introduction}

Intensity non-uniformity is the smooth intensity variation often seen in MR images caused by such factors as RF excitation field inhomogeneity [1], non-uniform reception coil sensitivity, eddy currents driven by field gradients [2], as well as electrodynamic interactions with the object often described as $\mathrm{RF}$ penetration and standing wave effects [3]. In modern MRI scanners these variations are often subtle enough that they are difficult to detect by visual inspection, however, they do affect automated image analysis techniques [4], particularly segmentation techniques that assume homogeneity of intensity within each tissue class.

While initial efforts at correcting intensity non-uniformity were based on physical models or external measurements of the field variations $[1,5]$, these methods are not sufficiently accurate to improve upon modern volumetric scans, such as those produced of the brain using a birdcage coil. Instead, recent efforts have focused on data driven strategies [6-9] based on statistical definitions of image uniformity. However, these methods are confounded by true anatomical variation that mimics the variations caused by intensity non-uniformity.

In this paper, we develop and validate, for a simple geometry, a physical model of intensity non-uniformity that has sufficient accuracy to predict the variations in a $1.5 \mathrm{~T}$ scanner using circularly polarized coils. While this geometry is too simple to accurately predict the variations in individual anatomical scans, it is sufficient to investigate a number of observations and hypotheses. (i) The widely made assumption that the data is corrupted by a smooth multiplicative field is accurate for proton density images. (ii) The pattern of intensity 
variation is highly dependent on the shape of the object being scanned. (iii) Elliptically shaped objects produce a diagonal pattern of variation when scanned using circularly polarized coils.

\section{Methods}

\subsection{Modelling the RF excitation field and reception sensitivity}

For simplicity, an analytic approach is used to investigate the effect of eccentric geometry on intensity non-uniformity. This treatment considers a long homogeneous dielectric cylinder with elliptic cross section excited by a circularly polarized field perpendicular to the cylinder axis. Interaction with the RF coil is neglected and far from the cylinder the excitation field $\left(B_{1}\right)$ is assumed to be uniform. The propagation of electric and magnetic fields in dielectric media is governed by the equations:

$$
\begin{aligned}
\nabla^{2} \mathbf{E} & =\mu \epsilon \frac{\partial^{2} \mathbf{E}}{\partial t^{2}}+\frac{\mu}{\rho} \frac{\partial \mathbf{E}}{\partial t} \\
\nabla \times \mathbf{E} & =-\frac{\partial \mathbf{B}}{\partial t}
\end{aligned}
$$

where $\mathbf{E}$ and $\mathbf{B}$ are the electric and magnetic fields respectively, $\mu$ is the magnetic permeability, $\epsilon$ is the permittivity, and $\rho$ is the resistivity of the media. The magnetic field $B_{1}$ is assumed to be oriented perpendicular to the cylinder axis while the electric field is oriented parallel. In addition, the solutions for the real vector fields $\mathbf{E}$ and $\mathbf{B}$ are assumed to vary sinusoidally in time at an angular frequency $\omega$ such that

$$
\begin{aligned}
& \mathbf{E}=\operatorname{Re}\left\{E_{z} \hat{z} e^{j \omega t}\right\} \\
& \mathbf{B}=\operatorname{Re}\left\{\left(B_{x} \hat{x}-j B_{y} \hat{y}\right) e^{j \omega t}\right\}
\end{aligned}
$$

Solutions to this problem for the cases of circular and elliptic cylinders are given in [10] and [11].

A so-called circularly polarized excitation field is created by driving two orthogonal linearly polarized coils $90^{\circ}$ out of phase such that the field components that contribute to the MR signal add constructively. In general, the field produced by a linearly polarized coil will vary in magnitude and direction within the object such that the combined field from the two coils can have arbitrary elliptic polarization. This elliptically polarized field can be decomposed into a $(+)$ rotating field, which causes the excitation, and a typically weaker $(-)$ counterrotating field that does not [12]. The orientation of this $(+)$ rotating field with respect to the driving field can be interpreted as a phase shift, which we refer to as a geometric phase shift.

In general, the individual field components will be complex to reflect the phase delays caused by currents induced in the object. Geometric and inductive 
phase shifts combine to determine the local phase of the excitation field within the object as follows:

$$
\mathrm{B}^{+}=B_{x x}+j B_{y x}-j B_{x y}+B_{y y} .
$$

where the notation $B_{y x}$ refers to the magnetic field in the $y$ direction produced by a coil aligned along the $x$ axis. While this derivation is based on two linearly polarized coils aligned with the $\mathrm{x}$ and $\mathrm{y}$ axes, the result is general for any combination of coils producing a circularly polarized field. Hence, it can used to predict the field pattern of a birdcage coil or a pair of linearly polarized coils not aligned with the $\mathrm{x}$ and $\mathrm{y}$ axes.

The same solutions for the field components apply when orthogonal coils are used for reception. However, the geometric phase shifts caused by reception cancel with those of the excitation field [1] whereas the phase shifts due to induced currents accumulate. Hence, the reception sensitivity is given by

$$
\mathrm{R}^{+}=R_{0}\left(B_{x x}-j B_{y x}+j B_{x y}+B_{y y}\right),
$$

where $R_{0}$ is a scale factor reflecting the sensitivity of the coil.

Images produced by a spin echo sequence are simulated using the derived excitation field and reception sensitivity. The signal measured for a spin echo pulse sequence is given by [10]

$$
\begin{aligned}
S & =\rho \mathrm{R}^{+} S_{S E} \\
S_{S E} & =\sin ^{3}\left(\frac{\pi}{2} \frac{\left|\mathrm{B}^{+}\right|}{B_{m}}\right) \exp \left(j \arg \left(\mathrm{B}^{+}\right)\right),
\end{aligned}
$$

where $\rho$ is the spin density, $S_{S E}$ is the emitted signal, $S$ is the measured signal, and $B_{m}$ is the nominal field strength needed to produce a $90^{\circ}$ flip-angle. This derivation neglects relaxation and assumes complete recovery of the magnetization between repetitions.

\subsection{Phantom studies}

To validate our theoretical model of intensity non-uniformity, we constructed two $40 \mathrm{~cm}$ long plastic cylindrical containers with elliptic and circular cross sections respectively. The circular cylinder has an inside diameter of $17.5 \mathrm{~cm}$, while the elliptic cylinder has major and minor diameters of $20 \mathrm{~cm}$ and $15 \mathrm{~cm}$. Each cylinder was filled with various concentrations of $\mathrm{NaCl}$ solutions made from deionized water.

The conductivity and permittivity of each solution was computed based on the concentration of $\mathrm{NaCl}$ using data from [13]. The quantities of $\mathrm{NaCl}$ were $1.38 \mathrm{~g} / \mathrm{L}, 2.83 \mathrm{~g} / \mathrm{L}$, and $58.2 \mathrm{~g} / \mathrm{L}$, or roughly $24 \mathrm{mM}, 48 \mathrm{mM}$, and $100 \mathrm{mM}$, producing resistivities of $4.0 \Omega \mathrm{m}, 2.0 \Omega \mathrm{m}$, and $1.0 \Omega \mathrm{m}$ respectively. These resistivities span the range typical of biological tissues [3] at frequencies around $64 \mathrm{MHz}$. At this frequency, the relative permittivity of water is essentially unchanged from its D.C. value of $\epsilon_{r}=80$, which is comparable to that of brain [14] at $64 \mathrm{MHz}$. 
In addition to $\mathrm{NaCl}$, a small quantity of $\mathrm{MnCl}_{2}$ was added to each solution to bring its concentration to $97 \mu \mathrm{M}$ so as to reduce $T_{1}$ relaxation times to approximately $910 \mathrm{~ms}$. Experiments using long repetition times (TR $=30 \mathrm{~s})$ showed no measurable change in intensity non-uniformity after addition of $\mathrm{MnCl}_{2}$.

For the experiments, the cylinders were aligned axially with the isocenter of the body coil of a 1.5T Siemens Vision MRI scanner and scanned transversally using a $B_{1}$ field mapping sequence [15] as well as a standard spin echo sequence. All images were acquired at $2 \mathrm{~mm}$ in-plane resolution and $6 \mathrm{~mm}$ slice thickness. The spin echo sequence ( $\mathrm{TR} / \mathrm{TE}=8 \mathrm{~s} / 14 \mathrm{~ms}$ ) had sufficiently short $\mathrm{TE}$ and long TR that relaxation can be neglected. The field mapping sequence is a stimulated echo technique $\left(90^{\circ}-\tau_{e} / 2-90^{\circ}-\tau_{1}-\alpha-\tau_{2}-90^{\circ}-\tau_{e} / 2-\right.$ acquire where $\tau_{e} / \tau_{1} / \tau_{2} / T R=36 \mathrm{~ms} / 60 \mathrm{~ms} / 8 \mathrm{~ms} / 1 \mathrm{~s}$ ) which yields a series of images whose intensities are related by

$$
S_{i}=a \cos b \alpha_{i} .
$$

The parameters $a$ and $b$ are computed at each voxel by a non-linear least squares fit to the flip angles $\alpha_{i}$ and complex image values $S_{i}$. Images were acquired at $\alpha=0^{\circ}, 40^{\circ}, \ldots, 400^{\circ}$. The resulting parameter map $b$ is proportional to the excitation field strength, while the parameter map $a$ is roughly proportional to spin density.

\section{Results}

\subsection{Simulated spin echo images}

Once the expressions for excitation field and reception sensitivity have been evaluated they can be used to simulate an imaging sequence. A simulated spin echo image for an elliptic geometry having $\mu=\mu_{0}, \epsilon_{r}=80$, and $\rho=2 \Omega m$ is shown in Figure 1. Also shown are the corresponding excitation field and reception sensitivity.

It should be noted that the pattern of non-uniformity in the spin echo image resembles neither the excitation field nor the reception sensitivity. This is caused by the apparent reversal of the excitation field to produce the sensitivity map. However, close inspection of the phase images for the two cases reveals that the excitation field and reception sensitivity maps differ by more than a reversal. In particular the geometric phase in the two cases is opposite while the inductive phase lag, dominant in this medium, remains unchanged.

Due to the symmetry of the elliptic shape, the magnitude of the excitation and reception sensitivity maps differ only by a reversal of the $y$ axis. However, the resulting spin echo image is not symmetric as reception sensitivity makes a stronger contribution to image non-uniformity than does excitation field variation.

\subsection{Comparison with phantom studies}

The experimental data admits two types of comparisons with the theoretical model: a direct comparison of the measured excitation field with that predicted, 
field magnitude contours

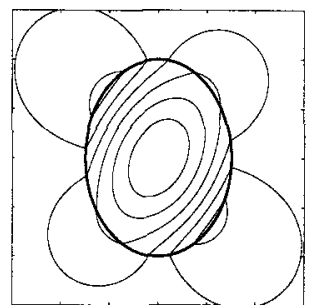

excitation field $\mathrm{B}^{+}$

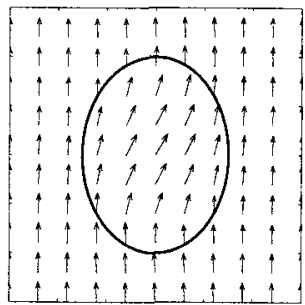

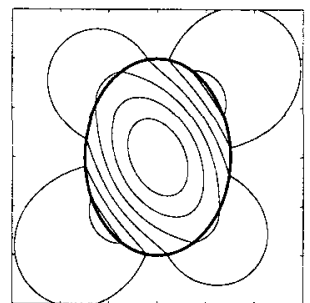

reception sensitivity $R^{+}$

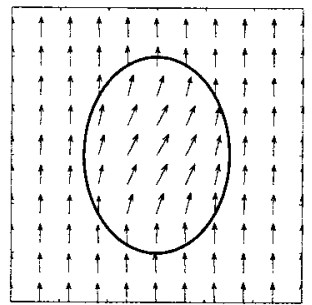

field direction vectors

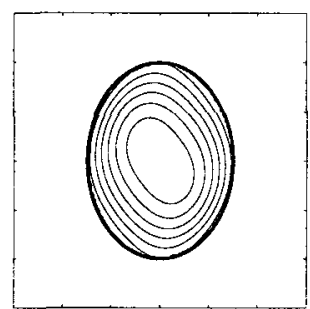

spin echo image

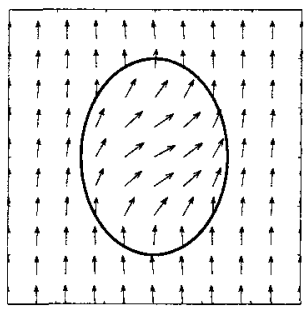

Fig. 1. Simulated spin echo images with nominal $90^{\circ}$ and $180^{\circ}$ flip-angles. Contours are at $5 \%$ of mean intensity. For the spin echo image the direction vectors can be interpreted as the phase of the complex image.

and a comparison of the measured spin echo image with that simulated from the predicted excitation field and reception sensitivity.

Before any comparisons were made all of the measured data was first automatically registered $[16,17]$ with the spin density map of the corresponding simulated image by linear transformation. A common mask was defined for each pair of images by automatically thresholding each image based on its histogram [18], taking the intersection of the two masks, and eroding it by $2 \mathrm{~mm}$. The RMS difference between two images was then computed within the common mask and expressed as a percentage of the mean intensity in the simulated image.

Figure 2 shows the excitation fields measured in the elliptic cylinder for each of the three $\mathrm{NaCl}$ solutions. Also shown are the predicted field patterns and the differences between the measured and predicted results. The prediction of a diagonal pattern of non-uniformity is confirmed by these experiments. When the gray scale of the difference image is expanded, it reveals spurious ghost images caused by the field mapping sequence as well as minor and largely random differences between the measured and predicted fields. The accuracy of the results for the circular cylinder is essentially the same.

The accuracy of the model at predicting the measured images was quantified by computing the root-mean-square (RMS) difference between the two. In all cases, the RMS difference was less than $2 \%$ and did not increase as the severity of the non-uniformity increased. 


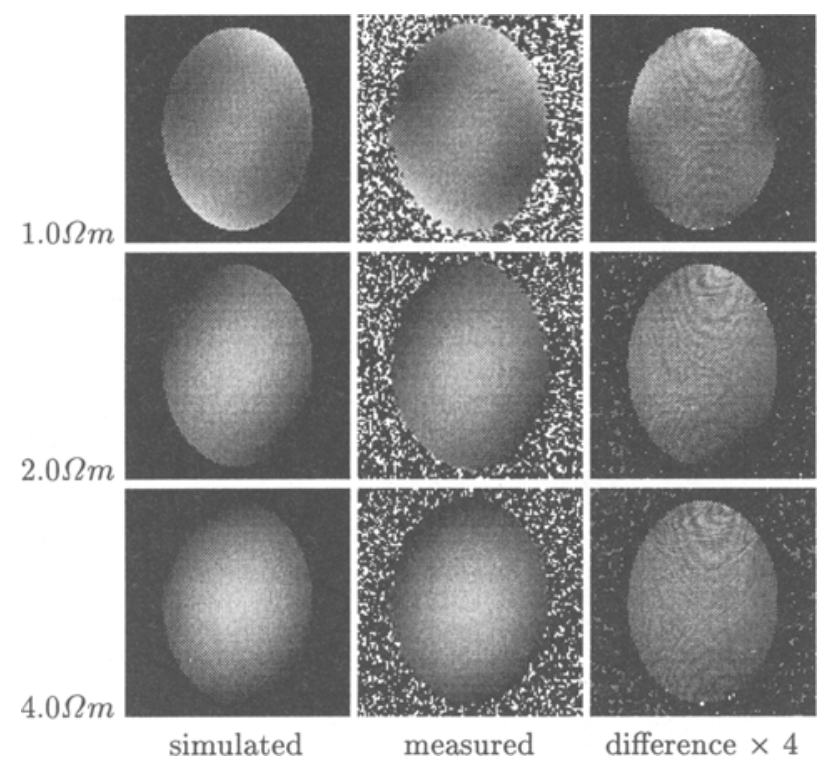

Fig. 2. Comparison of predicted and measured excitation fields $\mathrm{B}^{+}$in an elliptic phantom for three levels of resistivity. The normalized intensity scale for each image is 0.8 to 1.2 except for the difference images which are -0.05 to 0.05 .

Figure 3 shows the measured and predicted spin echo images for the two geometries and three solutions. The pattern of variations in these images is more complicated and the variations are more severe owing to the contribution of the reception sensitivity. Note that the orientation of the diagonal pattern in the elliptic case is reversed with respect to the excitation field map. The RMS difference between measured and predicted images was $1 \%-2 \%$.

\section{Discussion}

By modelling the electromagnetic interactions with the subject during excitation and reception from first principles, we are able to account for almost all of the intensity non-uniformity observed in volumetric scans at $1.5 \mathrm{~T}$. This agreement is achieved in spite of making no explicit assumptions of a coil producing the fields. While this is reasonable for a head sized object in a body coil, one can expect that smaller coils such as a head coil would produce some variations caused by their interaction with the object. However, in either case electromagnetic interaction with the object is the primary cause of intensity non-uniformity. Hence, the use of equation (7) is justified, and verifies that non-uniformity is correctly modelled as a smooth multiplicative field for proton density imaging sequences. However, for other imaging sequences that depend on relaxation, the 

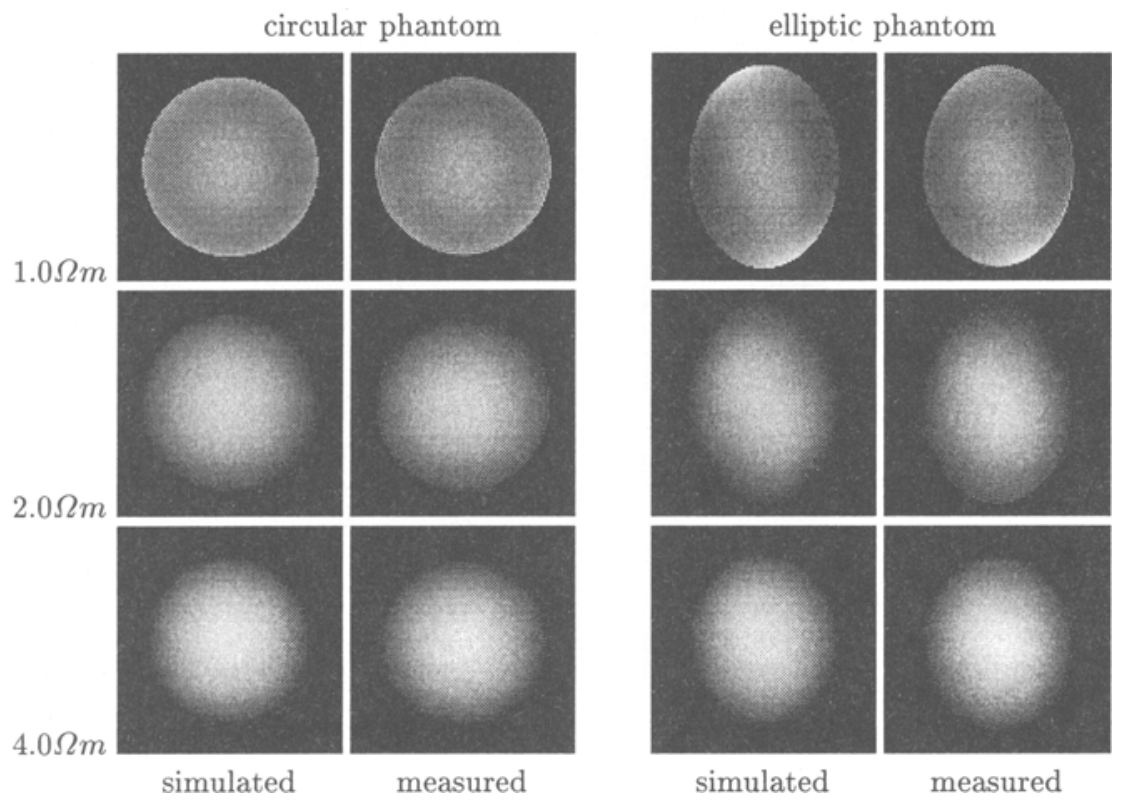

Fig. 3. Comparison of predicted and measured spin echo images for each level of resistivity. The normalized intensity scale for each image is 0.8 to 1.2.

term $S_{S E}$ in equation (7) will depend on the relaxation rates and will not in general be spatially smooth for inhomogeneous media.

We have shown that an elliptic geometry imaged using a circularly polarized coil produces a diagonal intensity non-uniformity pattern qualitatively similar to the quadrapole artifact observed with a linearly polarized coil. Although, one would expect the circularly symmetric pattern seen for circular objects to generalize to an elliptically symmetric pattern for elliptic objects, in the elliptic case the two linear fields interact with the media differently, leading to asymmetric intensity variations that do not cancel in the combined field. Such asymmetry could be incorrectly attributed to right-left hemisphere differences in a sensitive statistical analysis of neurological scans. Furthermore, since the orientation of this diagonal pattern is determined by the orientation of the object and not the orientation of the coil, one can expect that non-uniformity patterns will, in general, be correlated with anatomy and hence will also be present in averages of multiple scans.

Inspection of the experimental results for the elliptic case would suggest that the reception sensitivity is the mirror image of the excitation field pattern. However, the theoretical results show a more subtle relationship. In particular, for conductive objects there is a distinction between the phase delays associated with induced currents and those inherent in imaging the object in the absence of conductivity. Accounting for excitation and reception, the latter phase terms 
cancel while the former do not, thereby leading to the non-uniformity pattern in the spin echo image being more complicated than either the excitation or receptions fields.

An important implication of this lack of cancellation is that for arbitrarily shaped conductive objects, measurement of the excitation field is not sufficient to predict the pattern of variations in the resulting image. In addition, since electrodynamic interaction significantly affects the reception sensitivity, neither measurement of the sensitivity in the absence of the subject nor scanning of a standard phantom is sufficient to predict the variations in a given subject. Furthermore, these variations will be present irrespective of the uniformity of the field produced by the unloaded coil. As we have shown, the variation can be predicted by an electrodynamic model provided it takes into account the pulse sequence, geometry, and resistive and permittive properties of the subject.

\section{References}

1. E. R. McVeigh, M. J. Bronskill, and R. M. Henkelman, "Phase and sensitivity of receiver coils in magnetic resonance imaging," Med. Phys., vol. 13, pp. 806-814, Nov./Dec. 1986.

2. A. Simmons, P. S. Tofts, G. J. Barker, and S. R. Arridge, "Sources of intensity nonuniformity in spin echo images," Magn. Reson. Med., vol. 32, pp. 121-128, 1994.

3. P. A. Bottomley and E. R. Andrew, "RF magnetic field penetration, phase shift and power dissipation in biological tissue: implications for NMR imaging," Physics in Medicine and Biology, vol. 23, pp. 630-43, Jul 1978.

4. J. G. Sled and A. C. E. Alex P. Zijdenbos, D. Louis Collins, "The impact of intensity non-uniformity on automated anatomical analysis of 3d mri images," in Third International Conference on Functional Mapping of the Human Brain, p. S399, 1997.

5. B. R. Condon, J. Patterson, D. Wyper, et al., "Image non-uniformity in magnetic resonance imaging: its magnitude and methods for its correction," Br. J. Radiology, vol. 60 , pp. 83-87, 1987.

6. B. M. Dawant, A. P. Zijdenbos, and R. A. Margolin, "Correction of intensity variations in MR images for computer-aided tissue classification," IEEE Trans. Med. Imag., vol. 12, pp. 770-781, Dec. 1993.

7. C. R. Meyer, P. H. Bland, and J. Pipe, "Retrospective correction of intensity inhomogeneities in MRI," IEEE Transactions on Medical Imaging, vol. 14, pp. 3641, Mar. 1995.

8. W. M. Wells III, W. E. L. Grimson, R. Kikinis, and F. A. Jolesz, "Adaptive segmentation of MRI data," IEEE Trans. Med. Imag., vol. 15, no. 4, pp. 429-442, 1996.

9. J. G. Sled, A. P. Zijdenbos, and A. C. Evans, "A non-parametric method for automatic correction of intensity non-uniformity in MRI data," IEEE Trans. Med. Imag., vol. 17, pp. 87-97, February 1998.

10. G. O. Glover, C. E. Hayes, N. J. Pelc, W. A. Edelstein, O.M.Mueller, H. R. Hart, C. J. Hardy, M. O'Donnel, and W. D. Barber, "Comparison of linear and circular polarization for magnetic resonance imaging," J. Magn. Reson., vol. 64, pp. 255$270,1985$. 
11. J. G. Sled and G. B. Pike, "Standing-wave and RF penetration artifacts caused by elliptic geometry: an electrodynamic analysis of MRI," IEEE Trans. Med. Imag., 1997. (submitted).

12. P. S. Tofts, "Standing waves in uniform water phantoms," J. Magn. Reson. B, vol. 104, pp. 143-147, 1994.

13. P. S. Neelakanta, Handbook of electromagnetic materials: monolithic and composite versions and their applications, pp. 577-584. CRC Press, 1995.

14. D. Simunic, P. Wach, W. Renhart, and R. Stollberger, "Spatial distribution of high-frequency electromagnetic energy in human head during MRI: numerical results and measurements," IEEE Transactions on Biomedical Engineering, vol. 43, pp. 88-94, Jan 1996.

15. S. Topp, E. Adalsteinsson, and D. M. Spielman, "Fast multislice $B_{1}$-mapping," in International Society for Magnetic Resonance in Medicine, vol. 1, p. 281, 1997.

16. D. L. Collins, P. Neelin, T. M. Peters, and A. C. Evans, "Automatic 3D intersubject registration of MR volumetric data in standardized Talairach space," J. Comput. Assist. Tomogr., vol. 18, no. 2, pp. 192-205, 1994.

17. MNI Automated Linear Registration Package, Version 0.98, 1997. Available by anonymous ftp at $\mathrm{ftp}$ ://ftp.bic.mni.mcgill.ca/pub/mni_autoreg/

18. N. Otsu, "A threshold selection method from gray-level histograms," IEEE Transactions on Biomedical Engineering, vol. 9, pp. 63-66, 1979. 